\title{
Osteoporosis and Associated Factors among Thalassemia Patients Referred to Bone Mineral Density Screening -Dubai Hospital, 2014-2017
}

\author{
Massoumeh MH Zadeh ${ }^{*}$, Hamid Y Hussain ${ }^{2}$, Waleed Al Faisal ${ }^{2}$ and Muhammed Muhasin ${ }^{1}$ \\ ${ }^{1}$ Nuclear Medicine, Dubai Hospital, Dubai Health Authority, UAE \\ ${ }^{2}$ Primary Health Care Services Sector, Dubai Health Authority, UAE
}

\begin{abstract}
Background: Thalassemia is an inherited blood disorder of hemoglobin Synthesis long-term diseases, associated with reduced bone mineral density (BMD) and fractures due to many causes like hypogonadism, growth hormone deficiency, marrow expansion, and iron overload. Type of thalassemia and the treatment proved by many studies is associated with the bone diseases and recognized to be one of the main sources of related morbidities.

Objectives: To study the prevalence of osteoporosis among the thalassemia patients in Dubai hospital and to assess the role of some associated factors: Gender, Nationality, Age, Ferritin Value, Calcium and Vitamin D Level and baseline Hemoglobin.

Methodology: Records review study among a randomly selected systematic sample of a calculated sample size of about (280) at $95 \%$ confidence level. Using Open Epi (software) sample size was calculated from all cases referred to Dubai Hospital (nuclear medicine department) for osteoporosis screening of Bone mineral density (BMD) measurement. Several sensitive modalities and techniques are available for quantitative evaluation of the amount of total bone mass, but usage of dual X-ray absorptiometry (DEXA) of the lumbar spine, femoral neck suggested as one of the most consistent and non-invasive techniques for the assessment of bone mass. The non-invasive choice for repeated measurements of any temporal changes of BMD because of its $1 \%$ accuracy rate and low radiation exposure. Osteoporosis is "A disease characterized by low bone mass and micro- architectural deterioration of the bone tissue, leading to enhanced bone fragility and a consequent increase in fracture risk". The operational definition of variables been identified based on WHO criteria, inclusion-exclusion criteria were followed as all cases included except cases of osteoporosis due to other factors not related to thalassemia and repeated screening for same patient. Data collection questionnaires have been used for data collection, SPSS used for data entry, data analysis, level of significance is $95 \%$ and $\mathrm{P}$ value $<0.05$ was used.

Results: The study revealed that, about 280 patients referred nuclear medicine dept for osteoporosis assessment by DEXA test, to (136 Male, 144 Female) with thalassemia major 265 (94.6\%) and thalassemia intermedia 15(5.4\%), with mean age $23.1 \pm 7.5$ years and age range (11-45) years, mean BMI (21.7 \pm 4.6$)$, prevalence of osteoporosis and osteopenia in various parameters such as age group, Nationality, Gender and thalassemia type. As per our information, overall predominance rate of osteoporosis was $36.1 \%$ (101 out of 280) pediatrics 43 (42.6\%) adults 58 (57.4\%) whereas Prevalence rate in Osteopenia was $47.1 \%$ (132 out of 280$)$ pediatrics 53 (40.2\%), adults 79 (59.8\%). Normal BMD result reported 47 (16.8\%). Men prevalence rate of osteoporosis was 43 (31.6\%) while female incidence of osteoporosis was 58 (40.3\%). there was statistically significant association between patient's body mass index (BMI) and hemoglobin level with Osteoporosis while in patient with Osteopenia only nationality has statistically significant bond (Level of significant 0.05 ).
\end{abstract}

Conclusions: The rate of both osteoporosis and osteopenia is considerably high among thalamic group of population. Hemoglobin and body mass index were among the significant predictors for osteoporosis. Hemoglobin and body mass index management protocol need to be developed and applied among thalassemia cohorts to prevent osteoporotic pathological changes.

\section{Introduction}

Thalassemia is an inherited blood disorder of hemoglobin Synthesis long-term diseases [1]. Type of thalassemia as well as treatment proved by many studies to be associated with reduced bone mineral density (BMD) and fractures, therefore recognized to be one of the main sources of related morbidities. Osteopenia and osteoporosis are examples of bone disease among thalassemia patients [2,3]. Osteoporosis in thalassemia patients is particularly related to various risk factors, for instance, Duration of the diseases, Baseline Haemoglobin level, the onset of puberty, iron toxicity, hormonal disorders and nutritional deficiency $[4,5]$.

The total annual prevalence of symptomatic thalassemia is anticipated at 1 in 100,000 worldwide and 1 in 10,000 people in the Europe. Thalassemia is clinically presented in three clinical modules: thalassemia major, intermedia, and minor. Thalassemia major can be recognized easily among newborns under two years old as severe microcytic anemia, mild jaundice, and hepatosplenomegaly. Thalassemia intermedia have milder clinical findings and appear in older age. Thalassemia minor or carriers are usually asymptomatic but sometimes may have mild anemia [1]. As treatment with transfusion

Correspondence to: Massoumeh M.H. Zadeh, Nuclear Medicine, Dubai Hospital, Dubai Health Authority, UAE, E-mail: massum@gmail.com

Key words: osteoporosis, thalassemia, bone mineral density, screening, associated factors

Received: December 02, 2017; Accepted: December 20, 2017; Published: December 23, 2017 
programs and chelating therapy has significantly prolonged the survival of thalassemia patients, osteoporosis and osteopenia are common even in well-treated thalassemia patients $[2,6]$.

The International literature review showed that the prevalence of osteoporosis was $65.6 \%$ (214 out of 326 patients) [7]. The prevalence of osteoporosis to be 52- $96 \%$ among thalassemia patients Asian thalassemia population [8]. While the prevalence of fracture among thalassemia patient was $21.1 \%$ in North America [9]. The prevalence of osteoporosis in thalassemia Iraqi patients DXA scans was $67.5 \%$ while osteopenia 9.4\% and 22.9\% normal BMD reported [10].

This study is aiming to assess the occurrence of osteoporosis among thalassemia population in Dubai, which not been addressed so far. To answer the question, how common osteoporosis is among thalassemia patients in Dubai. Also what factors significantly associated with, as well as testing the hypothesis of presence or absence of some risk factors will have a true effect on the disease outcome in terms of emerging osteoporosis, which not been well assessed in Dubai.

\section{Objectives}

To study the prevalence of osteoporosis among thalassemia patients in Dubai and to assess the role of some associated factors (gender, nationality, baseline Hemoglobin).

\section{Methodology}

Retrospective records review study among a randomly selected systematic sample of calculated sample size using Open Epi Menu (software) for a sample size, simple random sample of 280, 136 (48.6\%) Male and $144(51.4 \%)$ Female at 95\% confidence interval, study samples are thalassemia patients (265 thalassemia major,15 thalassemia intermedia) referred to Dubai Hospital (nuclear medicine department) from August 2014 to September 2017, for osteoporosis screening by Bone mineral density (BMD) measurement. They had a mean age of $23.1 \pm 7.5$ years (range 11-45 years) with body mass index (BMI) of $21.7 \pm 4.6$ (Table 1). There are a number of sensitive modalities and methods for measurement of total bone mass. Dual X-ray absorptiometry (DEXA) of the lumbar spine and femoral neck is suggested as one of the most consistent and non-invasive procedures for the evaluation of bone mass [6,11]. DEXA is an outstanding noninvasive alternative for frequent evaluation of any chronological changes of BMD due to $1 \%$ accuracy rate and low radiation exposure [4]. The operational definition of variables has been identified based on WHO criteria (Osteoporosis is a disorder of skeletal insufficiency that is typically a result of increased bone breakdown relative to bone formation) [12]. Dual x-ray absorptiometry (The HOLOGIC Discovery DXA System) was utilized for all patients, both at the lumbar spine (L1-L4) and at the femoral neck. The system was aligned once a day as indicated by the manufacturer directions. The " $T$ score" and the " $Z$ score" were calculated at DEXA scan. The $T$ score is ideal parameter in adult above 20 years old. $T$ Score does not have clinical value in children and youth so $Z$ score is the chosen parameter in children below age 20 years old. According World Health Organization (WHO) definition of osteoporosis and diagnosis category, $T$ score of -1.0 or higher is considered normal. Osteopenia has been defined as a " $T$ score" between -1.0 and -2.5 while osteoporosis has been defined as score of -2.5 or lower [12]. Inclusion-exclusion criteria were followed as all cases included except cases of osteoporosis due to other factors not related to thalassemia. Structure questionnaires have been developed and coded for data collection including (socio-demographic data, clinical-related data, associated factors related data), questioner was validated by numbers of expert feedbacks, SPSS 20 software has been used for data entry, data analysis, level of significance is $95 \%$ and $P$ value $<0.05$ was used.

\section{Results}

This study revealed that, about 280 patients referred nuclear medicine department for DEXA assessment, to (136 Male, 144 Female) with thalassemia major $265(94.6 \%)$ and thalassemia intermedia $15(5.4 \%)$, with mean age $23.1 \pm 7.5$ years and age range (11-45) years, mean BMI $(21.7 \pm 4.6)$ as reflected in table 1 .

The study showed in table 2 represents prevalence of osteoporosis and osteopenia in various parameters such as age group, Nationality, Gender and thalassemia type. As per our information, overall predominance rate of osteoporosis was $36.1 \%$ (101 out of 280) pediatrics $43(42.6 \%)$ adults $58(57.4 \%)$ whereas Prevalence rate in Osteopenia was $47.1 \%$ (132 out of 280) pediatrics 53 (40.2\%), adults 79 (59.8\%). Normal BMD result reported 47 (16.8\%). Men prevalence rate of osteoporosis was 43 (31.6\%) while female incidence of osteoporosis was $58(40.3 \%)$.

The study reflected that by applying step wise logistic regression, there was statistically significant association between patient's body mass index (BMI) and hemoglobin level with Osteoporosis while in patient with Osteopenia only nationality has statistically significant bond. (Level of significant 0.05) (Table 3).

Table 1. Socio Demographic and biochemical characteristics of patients with Thalassemia major and intermedia

\begin{tabular}{|c|c|c|}
\hline \multicolumn{3}{|c|}{ Total } \\
\hline \multirow{2}{*}{ Age } & Mean \pm SD & $23.1 \pm 7.5$ \\
\hline & Median (range) & $23(11-45)$ \\
\hline \multirow{2}{*}{ Gender } & Male & $136(48.6 \%)$ \\
\hline & Female & $144(51.4 \%)$ \\
\hline \multirow{2}{*}{ Height } & Mean \pm SD & $157.8 \pm 9.8$ \\
\hline & Median (range) & $157(131-190)$ \\
\hline \multirow{2}{*}{ Weight } & Mean \pm SD & $54.5 \pm 13$ \\
\hline & Median (range) & $53(25-108)$ \\
\hline \multirow{2}{*}{ BMI } & Mean \pm SD & $21.7 \pm 4.6$ \\
\hline & Median (range) & $20.9(13.7-42.3)$ \\
\hline Ferritin (ng/ml) & Mean \pm SD & $2762 \pm 3066$ \\
\hline Calcium (mg/dL) & Mean \pm SD & $9.3 \pm 0.6$ \\
\hline Hemoglobin (g/dL) & Mean \pm SD & $9.9 \pm 1.3$ \\
\hline 25 OH Vitamin D & Mean \pm SD & $24.1 \pm 10.9$ \\
\hline
\end{tabular}

Table 2. Frequency distribution of osteoporosis and osteopenia according to socio demographic factors

\begin{tabular}{|c|c|c|c|c|c|}
\hline \multirow{2}{*}{ Overall } & \multicolumn{4}{|c|}{ BMD Result } \\
\cline { 3 - 6 } & & Osteoporosis & Osteopenia & $\begin{array}{c}\text { Normal } \\
\text { BMD }\end{array}$ & Total \\
\cline { 3 - 6 } & $101(36.1 \%)$ & $132(47.1 \%)$ & $47(16.8 \%)$ & 280 \\
\hline \multirow{3}{*}{ Age Group } & Pediatric & $43(42.6 \%)$ & $53(40.2 \%)$ & $22(46.8 \%)$ & $118(42.2 \%)$ \\
\cline { 2 - 6 } & Adult & $58(57.4 \%)$ & $79(59.8 \%)$ & $25(53.2 \%)$ & $162(57.8 \%)$ \\
\hline \multirow{3}{*}{ Nationality } & UAE & $21(21 \%)$ & $49(37 \%)$ & $12(26 \%)$ & $82(28 \%)$ \\
\cline { 2 - 6 } & Arab & $35(35 \%)$ & $32(24 \%)$ & $7(15 \%)$ & $74(26 \%)$ \\
\cline { 2 - 6 } & Indian & $6(6 \%)$ & $13(10 \%)$ & $3(6 \%)$ & $22(8 \%)$ \\
\cline { 2 - 6 } & Others & $39(38 \%)$ & $38(29 \%)$ & $25(53 \%)$ & $102(37 \%)$ \\
\hline \multirow{2}{*}{ Gender } & Male & $43(31.6 \%)$ & $63(46.4 \%)$ & $30(22 \%)$ & $136(49 \%)$ \\
\cline { 2 - 6 } & Female & $58(40.3 \%)$ & $69(48 \%)$ & $17(11.7 \%)$ & $144(51 \%)$ \\
\hline \multirow{2}{*}{ Thalassemia } & Major & $96(36.2 \%)$ & $125(44.6 \%)$ & $44(16.6 \%)$ & $265(94.6 \%)$ \\
\cline { 2 - 6 } & Intermedia & $5(33.3 \%)$ & $7(46.6 \%)$ & $3(20 \%)$ & $15(5.4 \%)$ \\
\hline
\end{tabular}




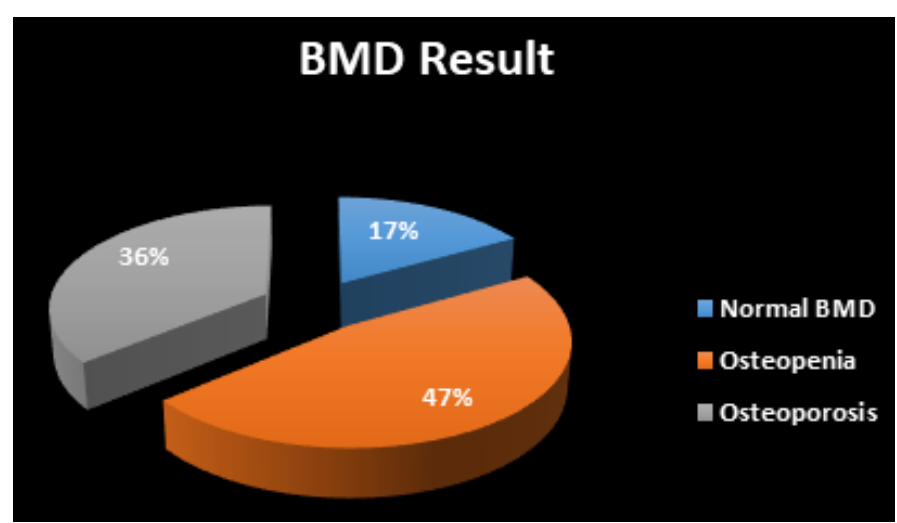

Figure 1. Frequency distribution of BMD result in Thalassemia major and intermedia patients

Table 3. Stepwise logistic of factors related to osteoporosis among thalassemic patient

\begin{tabular}{|c|c|c|}
\hline \multirow{2}{*}{ Variables } & \multicolumn{2}{|c|}{ P Value } \\
\cline { 2 - 3 } & Osteoporosis & Osteopenia \\
\hline Age & .160 & .652 \\
\hline Gender & .129 & .539 \\
\hline BMI & .000 & .229 \\
\hline Nationality & .350 & .017 \\
\hline Vit D & .460 & .203 \\
\hline Ferritin & .666 & .831 \\
\hline calcium & .907 & .348 \\
\hline Hb & .048 & .069 \\
\hline
\end{tabular}

\section{Discussions}

Current study shows that prevalence of osteoporosis and osteopenia among thalassemia major and intermedia who's referred to Dubai hospital is high (83.2\%). Incidence of Osteoporosis is $36.1 \%$ which is lower than Osteopenia $47.1 \%$ ). This finding is clearly different from other studies findings in the international literature that reported the prevalence of osteoporosis in Iran 65.6\% [7]. The prevalence of osteoporosis 52- 96\% among Asian thalassemia population [8], 21.1\% in North America [9] and in thalassemia Iraqi patients 67.5\% [10].

The explanation behind this assortment can be based on the nature of population in Dubai (UAE) which has diverse nationalities and, people from different countries backgrounds are living and working in Dubai, thus this study sample population is not homogenous one, and reflecting different identities.

Based on the current study findings, osteoporosis among adult thalassemia patient $(>20$ Years old) showed higher than childhood thalassemia patient group which can be explained on the bases of long term exposure to the thalassemia pathological process among adult comparing to children. More, osteoporosis's occurrence amongst Arab national (UAE and other Arab) proved to be higher compared to other nationalities which can be again explained by the spread of familial and genetic predisposition in the Arab Land east Mediterranean region). Osteoporosis exist more in female than male thalassemia patient may be due to the effect of feminizing hormones.

The interesting findings come out of the existing is that thalassemia patient with low BMI and low Hemoglobin level are at increased risk of osteoporosis. This can be due to the effect of low hematopoiesis process reflected by low Hemoglobin along with low bone minimization by low BMI and may be enhancing of osteolytic activities at bone structure. In order to avoid the effect of such pathogenesis, we should advice patients to keep their BMI and hemoglobin in normal level.

\section{Conclusions}

The progress rates of both osteoporosis and osteopenia changes are considerably high among thalassemia group of population, Hemoglobin and body mass index were among the significant predictors for osteoporosis.

\section{Recommendations}

Hemoglobin and body mass index management protocol needs to developed and applied among thalacemia cohorts to prevent osteoporotic pathological changes

\section{Acknowledgements}

I would like to express my very great appreciation to staffs of Nuclear Medicine Center in Dubai Hospital, Bhavna Ray and Ani K. Alexy for their assistance with the collection of data.

\section{Conflict of interest}

All Authors declaring that there is no conflict of interest.

\section{References}

1. Galanello R, Origa R (2010) Beta-thalassemia. Orphanet J Rare Dis 5: 11. [Crossref]

2. Vichinsky EP (1998) The morbidity of bone disease in thalassemia. Ann N Y Acad Sci 850: 344-348. [Crossref]

3. Cefalu CA (2004) Is bone mineral density predictive of fracture risk reduction? Cur Med Res Opin 20: 341-349. [Crossref]

4. Angelopoulos NG, Katounda E, Rombopoulos G, Goula A, Kaltzidou V, et al. (2006) Evaluation of bone mineral density of the lumbar spine in patients with beta-thalassemia major with dual-energy $\mathrm{x}$-ray absorptiometry and quantitative computed tomography: a comparison study. J Pediatr Hematol Oncol 28: 73-8. [Crossref]

5. Meropi T, Nicos S (2010) Osteoporosis Syndrome in Thalassaemia Major

6. Hajjar RR, Kamel HK (2004) Osteoporosis for the home care physician. Part 1: etiology and current diagnostic strategies. J Am Med Dir Assoc 5: 192-196. [Crossref]

7. Hashemieh M, Azarkeivan A, Radfar M, Saneifard H, Hosseini-Zijoud SM, et al (2014) Prevalence of Osteoporosis among Thalassemia Patients from Zafar Adult Thalassemia Clinic, Iran. IJBC 6: 143-148.

8. Bielinski BK, Darbyshire P, Mathers L, Boivin CM, Shaw NJ (2001) Bone density in the Asian thalassaemic population: a cross-sectional review. Acta Paediatr 90: 12621266. [Crossref]

9. Vogiatzi MG, Macklin EA, Fung EB, Vichinsky E, Olivieri N, et al. (2006) Giardinaa,Bone. Prevalence of fractures among the Thalassemia syndromes in North America. Bone 38: 571-575. [Crossref]

10. Salim M AL Jadir, Mohamed Z Jalal, Median F AL Ghreer, Mozahem S AL Hamdani, Wamid R AL Omaree (2012) Osteoporosis in Iraqi patients with thalassemia. Arthritis Res Ther 14.

11. (1994) Assessment of fracture risk and its application to screening for postmenopausa osteoporosis. Report of a WHO Study Group. World Health Organ Tech Rep Ser 843: 1-129. [Crossref]

12. (2003) Prevention and management of osteoporosis. Report of a WHO Scientific Group. Geneva, World Health Organization (WHO Technical Report Series, No.921.

13. Orvieto R, Leichter I, Rachmilewitz EA, Margulies JY (1992) Bone density, mineral content, and cortical index in patients with thalassemia major and the correlation to their bone fractures, blood transfusions, and treatment with desferrioxamine. Calcif Tissue Int 50: 397-9. [Crossref]

14. Jensen CE, Tuck SM, Agnew JE, Koneru S, Morris RW, et al. (1998) High prevalence of low bone mass in thalassaemia major. Br J Haematol 103: 911-915. [Crossref]

15. De Sanctis V, Soliman AT, Elsedfy H, Yassin M, Canatan D, et al. (2013) Osteoporosis in thalassemia major: an update and the I-CET 2013 recommendations for surveillance and treatment. Pediatr Endocrinol Rev 11: 167-180. [Crossref] 
16. Engkakul P, Mahachoklertwattana P, Jaovisidha S, Chuansumrit A, Poomthavorn P, et al. (2013) Unrecognized vertebral fractures in adolescents and young adults with thalassemia syndromes. J Pediatr Hematol Oncol 35: 212-217. [Crossref]

17. Soliman AT, Khalafallah H, Ashour R (2009) Growth and factors affecting it in thalassemia major. Hemoglobin 33: S116-126. [Crossref]

18. Cefalu CA (2004) Is bone mineral density predictive of fracture risk reduction? Curr Med Res Opin 20: 341-349. [Crossref]
19. Saffari F, Mahyar A, Jalilolgadr S (2012) Endocrine and metabolic disorders in $\hat{\mathrm{I}}^{2}$ thalassemiamajor patients. Caspian J Intern Med 3: 466-472. [Crossref]

20. Soliman A, De Sanctis V, Elsedfy H, Yassin M, Skordis N, et al. (2013) Growth hormone deficiency in adults with thalassemia: an overview and the I-CET recommendations. Georgian Med News 222: 79-88. [Crossref]

21. Wong P, Fuller PJ, Gillespie MT, Kartsogiannis V, Strauss BJ, et al. (2013) Thalassemia bone disease: the association between nephrolithiasis, bone mineral density, and fractures. Osteoporos Int 24: 1965-1971. [Crossref]

Copyright: (C2017 Zadeh MMH. This is an open-access article distributed under the terms of the Creative Commons Attribution License, which permits unrestricted use, distribution, and reproduction in any medium, provided the original author and source are credited. 